\title{
Forkhead-box A1 regulates tumor cell growth and predicts prognosis in colorectal cancer
}

\author{
YOUNG-LAN PARK* , SEUNG-HUN KIM* , SUN-YOUNG PARK, MIN-WOO JUNG, \\ SANG-YOON HA, JUNG-HO CHOI, DAE-SEONG MYUNG, SUNG-BUM CHO, \\ WAN-SIK LEE, HYUN-SOO KIM and YOUNG-EUN JOO \\ Department of Internal Medicine, Chonnam National University Medical School, \\ Gwangju 501-757, Republic of Korea
}

Received November 5, 2018; Accepted March 26, 2019

DOI: $10.3892 /$ ijo.2019.4771

\begin{abstract}
Forkhead box A1 (FOXA1) functions as a tumor suppressor gene or an oncogene in various types of cancer; however, the distinct function of FOXA1 in colorectal cancer is unclear. The present study aimed to evaluate whether FOXA1 affects the oncogenic behavior of colorectal cancer cells, and to investigate its prognostic value in colorectal cancer. The impact of FOXA1 on tumor cell behavior was investigated using small interfering RNA and the pcDNA6-myc vector in human colorectal cancer cell lines. To investigate the role of FOXA1 in the progression of human colorectal cancer, an immunohistochemical technique was used to localize FOXA1 protein in paraffin-embedded tissue blocks obtained from 403 patients with colorectal cancer. Tumor cell apoptosis and proliferation were evaluated using a terminal deoxynucleotidyl transferase-mediated dUTP nick-end labeling assay and Ki-67 immunohistochemical staining, respectively. FOXA1 knockdown inhibited tumor cell invasion in colorectal cancer cells, and induced apoptosis and cell cycle arrest. FOXA1 knockdown activated cleaved caspase-poly (ADP-ribose) polymerase, upregulated the expression of p53 upregulated modulator of apoptosis, and downregulated $\mathrm{BH} 3$ interacting domain death agonist and myeloid cell leukemia-1, leading to the induction of apoptosis. FOXA1 knockdown increased the phosphorylation level of signal transducer and activator of transcription-3. By contrast, these results were reversed following the overexpression of FOXA1. The overexpression of FOXA1 was associated with differentiation, lymphovascular invasion, advanced tumor stage, depth of invasion, lymph node metastasis
\end{abstract}

Correspondence to: Professor Young-Eun Joo, Department of Internal Medicine, Chonnam National University Medical School, 8 Hak-Dong, Dong-ku, Gwangju 501-757, Republic of Korea

E-mail: yejoo@chonnam.ac.kr

*Contributed equally

Key words: forkhead box A1, cell survival, apoptosis, prognosis, colorectal neoplasm and poor survival rate. The mean Ki-67 labeling index value of FOXA1-positive tumors was significantly higher than that of FOXA1-negative tumors. However, no significant association was observed between the expression of FOXA1 and the mean apoptotic index value. These results indicate that FOXA1 is associated with tumor progression via the modulation of tumor cell survival in human colorectal cancer.

\section{Introduction}

Colorectal cancer is one of the leading causes of cancer-associated morbidity and mortality worldwide. Despite recent advances in surgery and chemoradiotherapy for colorectal cancer, the prognosis of patients with advanced colorectal cancer remains poor (1-3). Therefore, a better understanding of the molecular biology of colorectal cancer progression will provide clinically applicable biomarkers for the reliable prediction of cancer progression and the identification of novel therapeutic targets (3).

Forkhead box A1 (FOXA1) is a transcription factor of the forkhead box gene superfamily necessary for the binding and activity of other transcriptional factors to chromatin, and serves critical roles in the development and differentiation of epithelial cells in several human organs $(4,5)$. Studies have shown that the expression of FOXA1 is associated with the development and progression of various types of cancer (6-9) and its functions may change according to specific types of cancer (6-9). FOXA1 acts as a tumor suppressor in various types of human cancer, including cancer of the breast, endometrium, bladder, liver and pancreas (10-17). However, FOXA1 induces aggressive behavior in lung, esophageal, prostate and thyroid cancer, implicating an oncogenic role (18-22). Further molecular mechanistic studies have revealed that FOXA1 promotes tumor progression by recruiting other transcription factors, and acts as a transcription factor in suppressing tumor development by directly regulating target gene expression (6-9). FOXA1 is also known to control the specificity of cancer cell types due to of the existence of unique FOXA1 targeting in each cancer cell type (9). However, the biological functions, underlying mechanisms and clinical significance of FOXA1 in human colorectal cancer remain to be fully elucidated. Previously, 
only one study has reported the oncogenic role of FOXA1 in human colorectal cancer (23).

The present study aimed to evaluate whether FOXA1 affects the oncogenic biological behavior of human colorectal cancer cells, to assess the expression of FOXA1 in human colorectal cancer tissues and to examine its association with clinicopathological features, including survival rate.

\section{Materials and methods}

Cell culture and materials. Human colorectal carcinoma cell lines (HCT116, CCL-247 ${ }^{\mathrm{TM}}$; Caco2, HTB-37 ${ }^{\mathrm{TM}}$; - SW480, CCL-228 ${ }^{\mathrm{TM}}$; HT29, HTB-38 ${ }^{\mathrm{TM}}$; DLD1, CCL-221 ${ }^{\mathrm{TM}}$; and COLO205, CCL-222 ${ }^{\mathrm{TM}}$ ) were obtained from the American Type Culture Collection (Manassas, VA, USA). The DKO1 (CVCL-9798) cell line was purchased from the ExPASy (Cellosaurus, Lausanne, Switzerland). All cell lines, excluding HCT116 cells, were cultured in Dulbecco's modified Eagle's medium (DMEM; Gibco, Thermo Fisher Scientific, Inc., Waltham, MA, USA) supplemented with $10 \%$ fetal bovine serum (FBS; Gibco, Thermo Fisher Scientific, Inc.) and $1 \%$ penicillin/streptomycin, whereas the HCT116 cells were cultured in McCoy's 5a Medium(WelGENE,Inc.,Daegu,Korea) supplemented with $10 \% \mathrm{FBS}$ and $1 \%$ penicillin/streptomycin at $37^{\circ} \mathrm{C}$ in a humidified atmosphere with $5 \% \mathrm{CO}_{2}$.

Gene transfection. FOXA1 cDNA was subcloned into the pcDNA6-myc vector (Invitrogen, Thermo Fisher Scientific, Inc.). The FOXA1 construction was verified by sequencing. FOXA1 small interfering (si)RNA (GGAGGAGAGAUAAG UUAUA-dTdT) and scrambled siRNA (AllStars Negative Control siRNA, cat. no. 1027281) were purchased from Bioneer (Daejeon, Korea) and Qiagen GmbH (Hilden, Germany), respectively. To transfect siRNA, the SW480 and HCT116 cells were seeded into 6 -well plates at a density of $3 \times 10^{5}$ cells/well at $37^{\circ} \mathrm{C}$ and were $40-60 \%$ confluent at the time of transfection. For FOXA1 knockdown, $20 \mu \mathrm{M}$ of FOXA1 siRNA (FS) was transfected with $5 \mu$ l Lipofectamine $^{\mathrm{TM}}$ RNAiMAX reagent (Invitrogen; Thermo Fisher Scientific, Inc.). To overexpress FOXA1, $0.5 \mu \mathrm{g}$ of FOXO1-pcDNA6-myc vector $(\mathrm{FV})$ was transfected with $5 \mu$ l Lipofectamine ${ }^{\mathrm{TM}} 2000$ reagent (Invitrogen, Thermo Fisher Scientific, Inc.). Scrambled siRNA (SS) and empty-pcDNA6-myc vector (EV) were used as a negative control, respectively. Following incubation for $24 \mathrm{~h}$ at $37^{\circ} \mathrm{C}$, identification of the expression of FOXA1 was performed by western blotting and the transfected cells were used in the following experiments.

Cell proliferation assay. The transfected HCT116 and DLD1 cells were seeded into a 96-well plate at a density of $1 \times 10^{4}$ cells/well and incubated for $24 \mathrm{~h}$ at $37^{\circ} \mathrm{C}$. Cell viability was determined using a water-soluble tetrazolium salt (DoGen, Daeillab, Seoul, Korea). Following application, the absorbance at $450 \mathrm{~nm}$ was measured on a microplate reader (Infinite M200; Tecan, Mannedorf, Switzerland). Each experiment was performed in triplicate wells and was repeated at least three times.

Apoptosis analysis. The DLD1 and HCT116 cells at a density of $5 \times 10^{5} /$ well were seeded into a 6 -well plate and incubated for $24 \mathrm{~h}$ at $37^{\circ} \mathrm{C}$ prior to FOXA1 transfection. The transfected cells were collected and resuspended in $100 \mu \mathrm{l}$ of binding buffer (BD Biosciences, San Diego, CA, USA) for $20 \mathrm{~min}$ at $4^{\circ} \mathrm{C}$. The cells were incubated with 7-amino-actinomycin D (BD Biosciences) and Annexin V-APC (BD Biosciences) for 20 min at room temperature. To analyze the number of apoptotic cells, a FACSCalibur flow cytometer (BD Biosciences) and WinMDI version 2.9 (The Scripps Research Institute, San Diego, CA, USA) were used.

Cell cycle analysis. The DLD1 and HCT116 cells at a density of $5 \times 10^{5} /$ well were seeded into a 6 -well plate and transfected with FS and FV for $24 \mathrm{~h}$ at $37^{\circ} \mathrm{C}$. The transfected cells were fixed in ice-cold $70 \%$ ethanol for $1 \mathrm{~h}$ to determine the cell cycle distribution and then washed twice with PBS. The cells were incubated in $100 \mu \mathrm{l}$ of $10 \mu \mathrm{g} / \mathrm{ml}$ ribonuclease A (Sigma-Aldrich; Merck KGaA, Darmstadt, Germany) for $20 \mathrm{~min}$ at $37^{\circ} \mathrm{C}$. Following this, $100 \mu \mathrm{l}$ of $50 \mu \mathrm{g} / \mathrm{ml}$ propidium iodide (Sigma-Aldrich; Merck KGaA) was added for $30 \mathrm{~min}$ at room temperature in the dark. Cell cycle analysis was performed using a FACSCalibur flow cytometer (BD Biosciences) and WinMDI version 2.9 (The Scripps Research Institute).

Western blotting. The transfected cells were lysed in RIPA buffer containing Halt ${ }^{\mathrm{TM}}$ Protease inhibitor and Halt ${ }^{\mathrm{TM}}$ Phosphatase inhibitor cocktail (Thermo Fisher Scientific, Inc.) for $30 \mathrm{~min}$ in an ice bath. The protein concentration of the lysate from transfected cells was measured using the $\mathrm{BCA}^{\mathrm{TM}}$ protein assay (Thermo Fisher Scientific, Inc.). The proteins (20 $\mu \mathrm{g}$ per lane) were separated using 10\% sodium dodecyl sulfate-polyacrylamide gel electrophoresis and transferred onto a polyvinylidene fluoride (PVDF) membrane (Bio-Rad Laboratories, Inc., Hercules, CA, USA). The transferred membranes was blocked with $5 \%$ bovine serum albumin (BSA) for $1 \mathrm{~h}$ at room temperature and then incubated overnight at $4{ }^{\circ} \mathrm{C}$ with primary antibodies at 1:1,000 dilution. Antibodies against the following proteins were used: FOXA1 (cat. no. ab170933, Abcam, Cambridge, UK), cleaved poly (ADP-ribose) polymerase (PARP, cat. no. 5625), p53 upregulated modulator of apoptosis (PUMA; cat. no. 4976), Bax (cat. no. 2772), BH3 interacting domain death agonist (Bid; cat. no. 2002), Bcl-xL (cat. no. 2764), cyclin D1 (cat. no. 2926), p21 (cat. no. 2947), cyclin-dependent kinase 2 (CDK2, cat. no. 2546), CDK4 (cat. no. 2906), myeloid cell leukemia-1 (Mcl-1; cat. no. 5453), phospho-signal transducer and activator of transcription-3 (phospho-STAT3, cat. no. 9145), STAT3 (cat. no. 9139) from Cell Signaling Technology, Inc. (Danvers, MA, USA), and glyceraldehyde 3-phosphate dehydrogenase (GAPDH, cat. no. FL-335) from Santa Cruz Biotechnology, Inc.. The membranes were washed four times with Tris-buffered saline-0.1\% Tween-20 (TBS-T) and were incubated with a horseradish peroxidase-conjugated secondary antibody (anti-rabbit, cat. no. 7074, anti-mouse, cat. no. 7076, Cell Signaling, Technology, Inc.) at 1:2,000 dilution for $1 \mathrm{~h}$ at room temperature. Following washing with TBS-T, the protein bands were developed using an Enhanced Chemiluminescent reagent (Amersham, GE Healthcare Life Sciences) and were analyzed on the luminescence image analyzer LAS-4000 (Fujifilm, Tokyo, Japan). The bands of the immunoblot were quantified using Multi-Gauge software (ver. 3.0, Fujifilm). 
Transwell invasion assay. The transfected cells were plated in the upper well of Transwell filter chambers $(8.0-\mu \mathrm{m}$ pore size; Costar, Cambridge, MA, USA) coated with $1 \%$ gelatin. Fibronectin (10 $\mu \mathrm{g} / \mathrm{ml}$, Sigma-Aldrich; Merck KGaA) was added as a chemoattractant to $0.2 \%$ BSA (Sigma-Aldrich; Merck $\mathrm{KGaA}$ ) containing medium in the lower chamber. Following overnight incubation at $37^{\circ} \mathrm{C}$, the cells invaded to the lower surface of the upper chamber were fixed with $70 \%$ ethanol for $1 \mathrm{~min}$ at room temperature and stained with Hemacolor ${ }^{\circledR}$ Rapid staining solution (Merck KGaA) following the manufacturer's protocol. Under a light microscope (magnification, x200, Olympus BX51, Olympus, Tokyo, Japan), the stained upper chambers were placed on the corner squares of a hemocytometer and images were captured. Subsequently, the stained cells were counted in five selected fields (each $0.25 \mathrm{~mm}^{2}$, four smaller squares). The results are expressed as the mean $\pm \mathrm{SE}$ of the number of cells/field of three individual experiments.

Patients and tissue samples. Formalin-fixed, paraffin-embedded tissue blocks were obtained from 403 patients who underwent surgery for colorectal cancer at Chonnam National University Hwasun Hospital (Jeonnam, Korea) between July 2004 and June 2006. The tissue blocks were selected by viewing original pathologic slides and selecting blocks that showed the junction between normal colorectal epithelium and tumor tissue. Patient characteristics, including sex, age at the time of surgery, histologic grade and stage, were obtained by examining medical records and contacting pathologists and physicians when necessary. No patient had received anticancer therapy prior to surgery. The tumors were staged in accordance with the American Joint Committee on Cancer staging system (24). Overall survival was measured from the time of surgery until follow-up on December 31, 2013. The present study was approved by the Institutional Review Board of the Chonnam National University Hwasun Hospital (Jeonnam, Korea; CNUHH-2017-163). This was a retrospective study and written informed consent was obtained from each participant prior to tissue acquisition at the time of hospitalization. All participants provided written consent for their information to be stored in the hospital database and used for research.

Immunohistochemistry and evaluation of the expression of FOXA1. The paraffin-embedded tissue samples $(4-\mu \mathrm{m}$-thick tissue sections) were deparaffinized using xylene and rehydrated with ethanol at graded concentrations (100-60\%). The samples were then boiled in citrate buffer $(\mathrm{pH}$ 6.0, Dako, Agilent Technologies, Inc., Carpentaria, CA, USA) for $10 \mathrm{~min}$ in a pressure boiler for antigen retrieval. Endogenous peroxidase activity was blocked using peroxidase-blocking solution (Dako, Agilent Technologies, Inc.) for $10 \mathrm{~min}$ at room temperature, and non-specific reactivity was blocked with Dako ${ }^{\circledR}$ Protein Block Serum-Free solution (Dako, Agilent Technologies, Inc.) for $30 \mathrm{~min}$ at room temperature. The samples were incubated with primary anti-FOXA1 (1:200 dilution; cat. no. ab170933, Abcam) antibodies for $1 \mathrm{~h}$ at room temperature. Bound antibody was visualized with the DakoReal ${ }^{\mathrm{TM}}$ Envision HRP/DAB detection system (Dako, Agilent Technologies, Inc.). Nuclear counterstaining was performed with Mayer's hematoxylin solution (Sigma-Aldrich, Merck KGaA). The stained tissues were observed and images were captured under a light microscope (Olympus BX51). Immunohistochemical staining was assessed by two independent pathologists blinded to the patient clinical outcome data. FOXA1 staining intensity was scored as follows: 0, no staining; 1, weak; 2, moderate; 3 , strong. The percentage of the stained area was scored as 0 for the absence of positive staining in tumor cells, 1 for positive staining in $<10 \%$ of the tumor cells, 2 for positive staining in $10-50 \%$ of the tumor cells, and 3 for positive staining in $>50 \%$ of the tumor cells. The final score index was obtained by multiplying the intensity and area percentage scores. The final scores for the 403 tumor samples ranged between 0.0 and 12.0 with a mean of 6.0. Samples with a total score of $\geq 6$ were designated as positive for FOXA1 expression, whereas those with a total score of $<6$ were designated as negative for FOXA1 expression.

Assessment of apoptosis and tumor cellproliferation. Tumorcell apoptosis was determined using the terminal deoxynucleotidyl transferase-mediated dUTP nick-end labeling (TUNEL) system (Promega, Madison, MA, USA) according to the manufacturer's instructions. Briefly, the tissues were deparaffinized, rehydrated through a graded alcohol series (100-60\%) and incubated in permeabilization solution. Labeling was performed by adding the terminal deoxynucleotide transferase enzyme reaction mix to tissue sections mounted on slides. Following washing with TBS-T, the slides were incubated with the enzyme substrate 3,3-diaminobenzidine (DAB) for color development, which was used to localize the labeled cells. The apoptotic index (AI) was calculated as the number of TUNEL-positive cells per 1,000 tumor cell nuclei. Proliferation of the tumor cells was visualized by immunohistochemical staining with Ki-67 (1:500 dilution, cat. no. ab833, Abcam) antibody. The samples were incubated with primary anti-ki-67 diluent for $1 \mathrm{~h}$ at room temperature. Bound antibody was visualized with the DakoReal ${ }^{\mathrm{TM}}$ Envision HRP/DAB detection system (Dako, Agilent Technologies, Inc.). Nuclear counterstaining was performed with Mayer's hematoxylin solution (Sigma-Aldrich, Merck KGaA). The stained tissues were observed and images were captured under a light microscope (Olympus BX51). Nuclei stained with Ki-67 antibody were considered positive. The Ki-67 labeling index (KI) was defined as the number of Ki-67-positive nuclei per 1,000 tumor cell nuclei.

Statistical analysis. Statistical analyses were conducted using the Statistical Package for Social Sciences software version 20.0 (IBM Corp., Armonk, NY, USA). A $\chi^{2}$ test was used to analyze the association between the expression of FOXA1 and clinicopathological parameters. The survival rates were calculated using the Kaplan-Meier method, and the statistical significance of differences was examined using the log-rank test. The Student's t-test was used for the analysis of association between the expression of FOXA1 and the apoptosis and proliferation of cells in human colorectal cancer. Experimental differences between the FOXA1 knockdown or overexpression group and control group were tested with the Student's t-test. Each experiment was repeated at least three times. $\mathrm{P}<0.05$ was considered to indicate a statistically significant difference. 
A
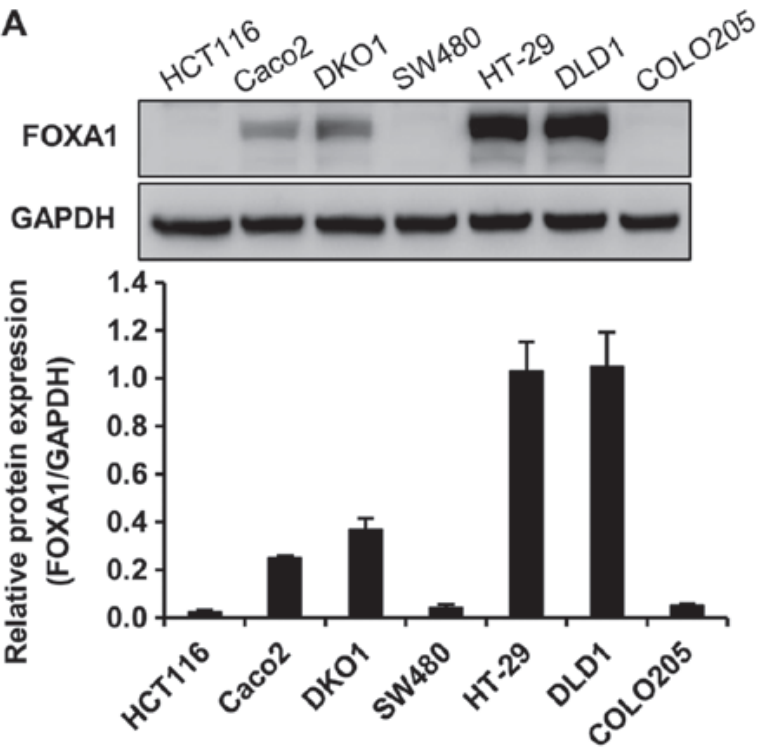

B
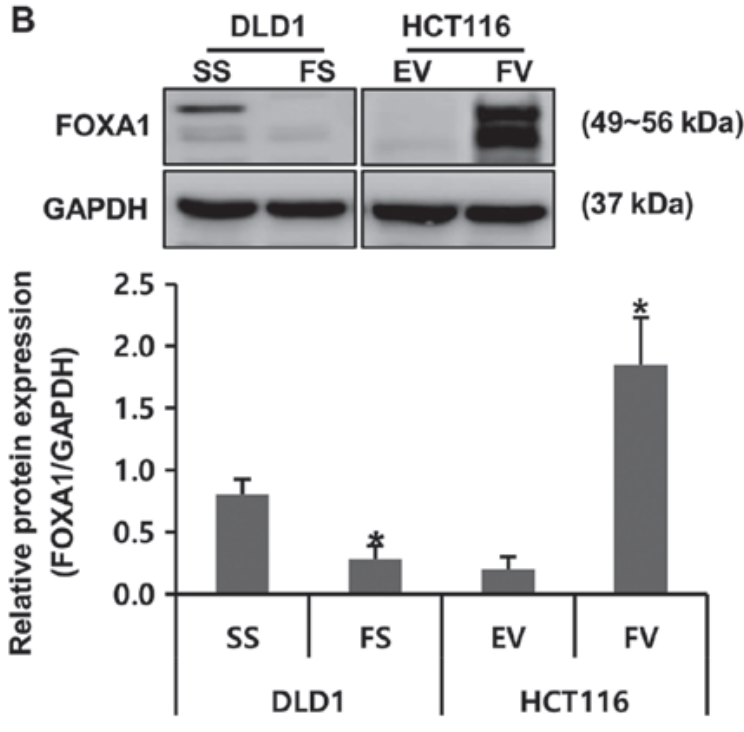

Figure 1. Protein expression of FOXA1 in human colorectal cancer cells. (A) Endogenous expression of FOXA1 protein in various human colorectal cancer cell lines, examined by western blot analysis. GAPDH was used as a loading control. (B) Knockdown and overexpression of FOXA1 protein was induced using FOXA1 siRNA and pcDNA6-myc-FOXA1, respectively ( $\mathrm{P}<0.05$, compared to respective control). Bands of the immunoblot were quantified using Multi-Gauge software (ver. 3.0). FOXA1, forkhead box protein A1; SS, scramble siRNA; FS, FOXA1 siRNA; EV, empty-pcDNA6-myc vector; FV, pcDNA6-myc-FOXA1 vector.
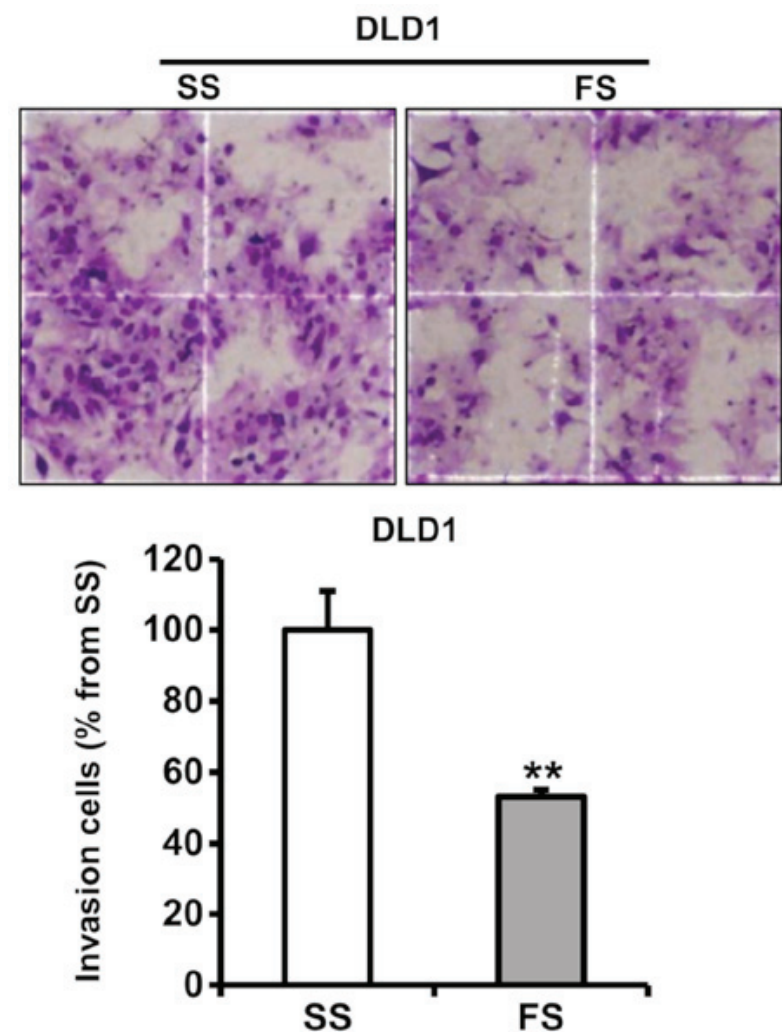

HCT116

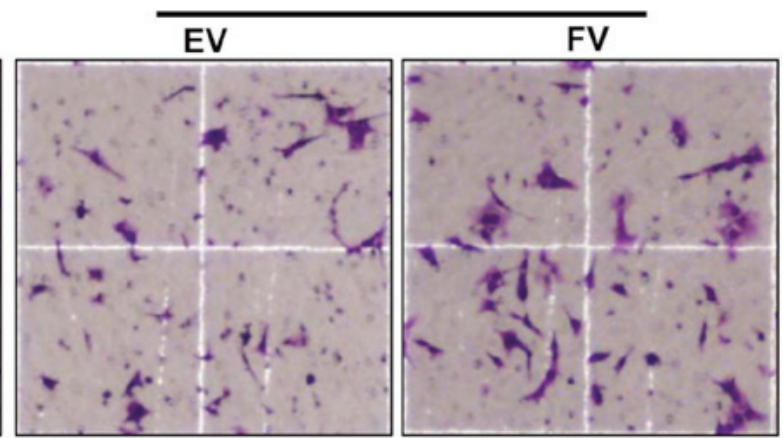

HCT116

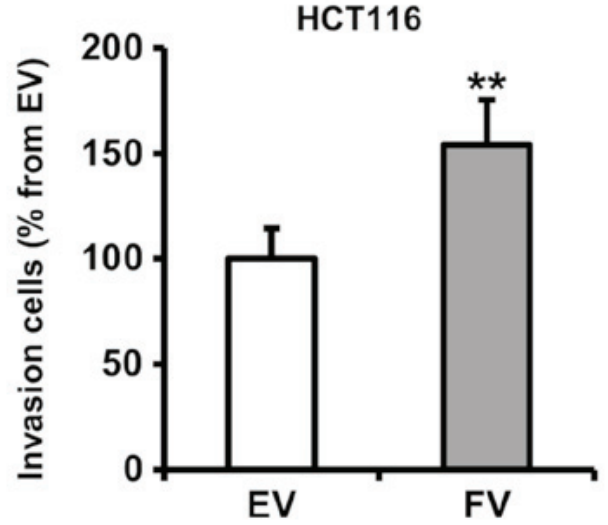

Figure 2. Impact of FOXA1 on the invasion of human colorectal cancer cells. An invasion assay using cells transfected with pcDNA6-myc or siRNA was performed. Stained invading cells were counted under a light microscope (magnification, $\mathrm{x} 200$, four smaller squares $=0.25 \mathrm{~mm} 2$ ) and are represented as a graph between groups. The number of FS-transfected cells that invaded was significantly lower than that of invading SS-transfected cells (mean \pm SE, $\mathrm{n}=6$; ${ }^{* *} \mathrm{P}<0.01$, compared to respective control). FOXA1, forkhead box protein A1; siRNA, small interfering RNA; SS, scrambled siRNA; FS, FOXA1 siRNA; EV, empty-pcDNA6-myc vector; FV, pcDNA6-myc-FOXA1 vector.

\section{Results}

Expression of FOXA1 in various human colorectal cancer cell lines. To investigate the protein expression of FOXA1 in colorectal cancer cells, various human colorectal cancer cell lines, including HCT116, DLD1, DKO1, SW480, Caco2, COLO205 and HT-29, were subjected to western blot analysis. Among these cells, the protein expression of FOXA1 was the 
A

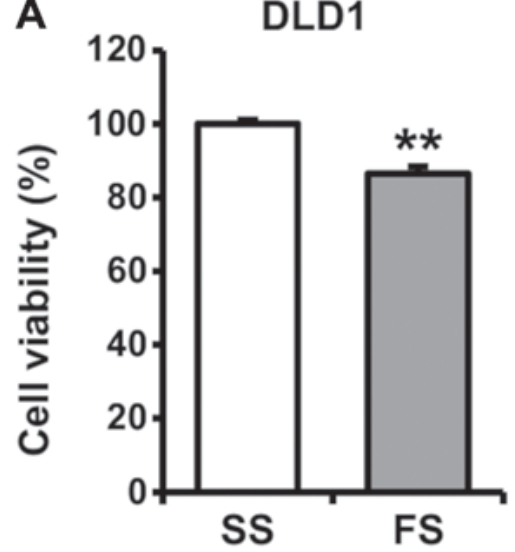

B

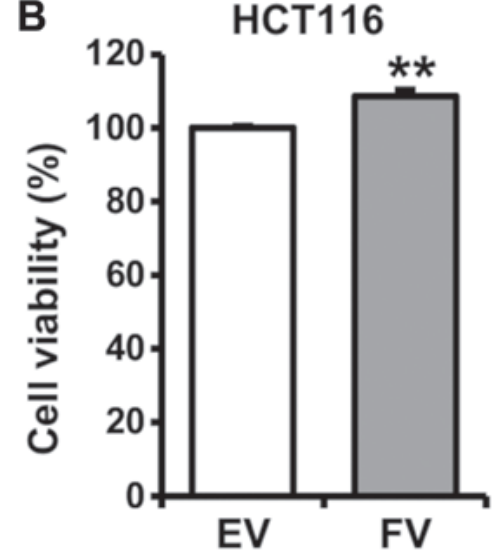

Figure 3. Impact of FOXA1 on the proliferation of human colorectal cancer cells. To determine the potential effects of FOXA1 on cell proliferation, cell viability was measured using the water-soluble tetrazolium salt assay. A cell proliferation assay was performed 2 days after transfection with pcDNA6-myc or siRNA. (A) Proliferating cells, as determined by absorbance, decreased significantly in the FS-transfected cells, compared with those in the SS-transfected DLD1 cells $\left({ }^{* *} \mathrm{P}<0.05\right)$. (B) FV-transfected HCT116 cells exhibited significantly increased proliferation compared with the EV-transfected cells $\left({ }^{* *} \mathrm{P}<0.05\right)$. FOXA1, forkhead box protein A1; siRNA, small interfering RNA; SS, scrambled siRNA; FS, FOXA1 siRNA; EV, empty-pcDNA6-myc vector; FV, pcDNA6-myc-FOXA1 vector.

highest in DLD1 cells and the lowest in HCT116 cells (Fig. 1A). FS or FV were used to modulate the endogenous protein expression of FOXA1 in DLD1 and HCT116 cells, respectively. The protein expression of FOXA1 was specifically decreased at the protein level by transfection of FS in DLD1 cells and was increased by the transfection of FV in HCT116 cells (Fig. 1B).

Impact of FOXA1 on the invasion of human colorectal cancer cells. The number of invading FS-transfected DLD1 cells was significantly decreased, compared with those in the SS-transfected cells $(\mathrm{P}<0.05)$. By contrast, the FV-transfected HCT116 cells exhibited a significantly increased number of invading cells compared with the EV-transfected cells $(\mathrm{P}<0.05)$ (Fig. 2).

Impact of FOXAl on human colorectal cancer cell proliferation. To determine the potential effects of FOXA1 on cell proliferation, cells were subjected to a cell proliferation assay 2 days after transfection with FS or FV. Proliferating cells, as determined by absorbance, were decreased significantly in the FS-transfected cells, compared with the SS-transfected cells in DLD1 cells $(\mathrm{P}<0.05)$. By contrast, the FV-transfected HCT116 cells exhibited significantly increased proliferation, compared with the EV-transfected cells $(\mathrm{P}<0.05)$ (Fig. 3).

Impact of FOXA1 on apoptosis and cell cycle distribution in human colorectal cancer cells. Flow cytometric analyses were performed to evaluate the impact of FOXA1 on apoptosis and cell cycle distribution. The apoptotic rate of cells transfected with FS was significantly increased, compared with those transfected with SS in DLD1 cells $(8.65 \pm 4.9$, vs. 12.69 $5.0 \%$; $\mathrm{P}<0.01)$. In addition, the apoptotic rate was decreased in HCT116 cells following the overexpression of FOXA1 (15.14 \pm 2.3 , vs. $13.89 \pm 0.2 \%$; Fig. 4A). To determine the activation of caspases during the knockdown and overexpression of FOXA1, caspase-specific activities were detected. The expression of cleaved PARP was increased in DLD1 cells following the knockdown of FOXA1, and was decreased in HCT116 cells following the overexpression of FOXA1 (Fig. 4B). Whether the impact of FOXA1 on apoptosis is associated with the modulation of apoptosis regulatory proteins was further examined. As shown in Fig. 4B, FOXA1 knockdown led to an increase in the pro-apoptotic protein, PUMA. By contrast, the overexpression of FOXA1 led to a decrease in PUMA and an increase in anti-apoptotic proteins Bid and Mcl-1. The effects of FOXA1 on cell cycle distribution and the regulators involved in cell cycle distribution were evaluated in human colorectal cancer cells. The overexpression of FOXA1 inhibited cell cycle arrest in the subG1 phase of HCT116 cells, and its knockdown induced cell cycle arrest in DLD1 cells (Fig. 5A). As shown in Fig. 5B, positive regulators of the cell cycle, including cyclin D1, CDK2 and CDK4, exhibited significantly decreased protein levels, whereas the negative regulator of CDKI-p21 was significantly increased by FOXA1 knockdown in DLD1 cells. The protein levels of cyclin D1 and CDK2 were significantly increased and the protein level of p21 was significantly decreased by the overexpression of FOXA1 in HCT116 cells (Fig. 5B).

Impact of FOXA1 on the oncogenic signaling pathway in human colorectal cancer cells. To examine whether FOXA1 activates oncogenic signaling pathways in human colorectal cancer cells, the present study determined the phosphorylation level of STAT3 signaling protein using western blotting. It was found that the phosphorylation of STAT3 was decreased by FOXA1 knockdown in DLD1 cells. By contrast, the phosphorylation of STAT3 was increased by the overexpression of FOXA1 in HCT116 cells (Fig. 6).

Association between FOXA1 and clinicopathological parameters of human colorectal cancer. To examine the prognostic role of FOXA1 in the progression of human colorectal cancer, the present study investigated the expression of FOXA1 immunohistochemically in formalin-fixed, paraffin-embedded tissue sections obtained from 403 patients with colorectal cancer, and results were associated with the clinicopathological data of these patients. FOXA1 protein 
A

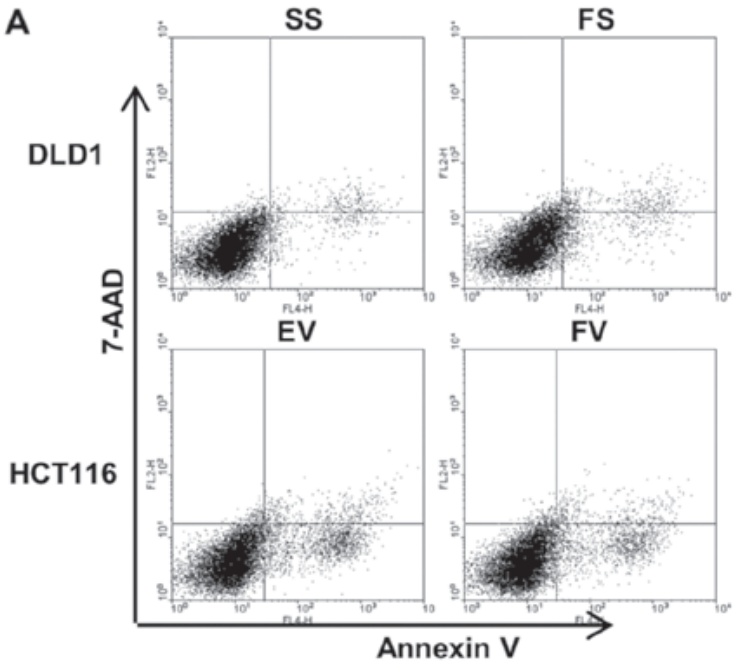

B

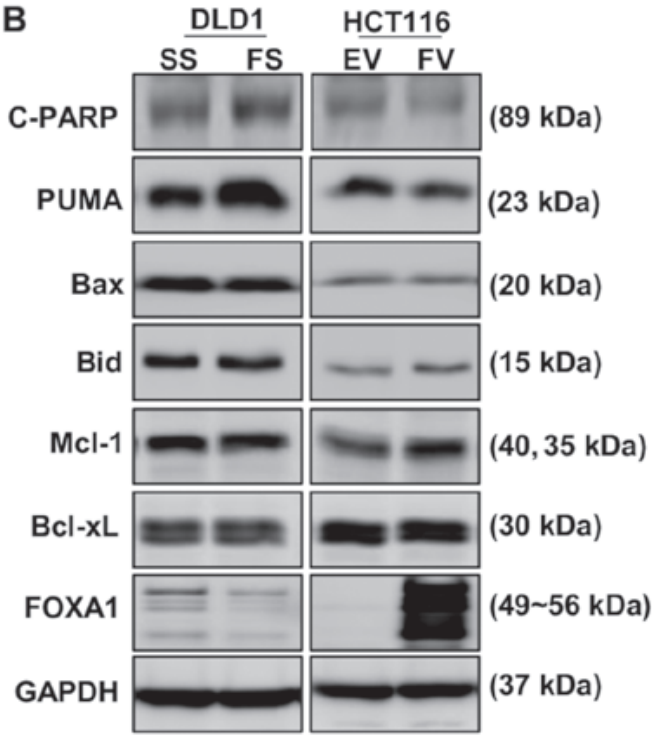

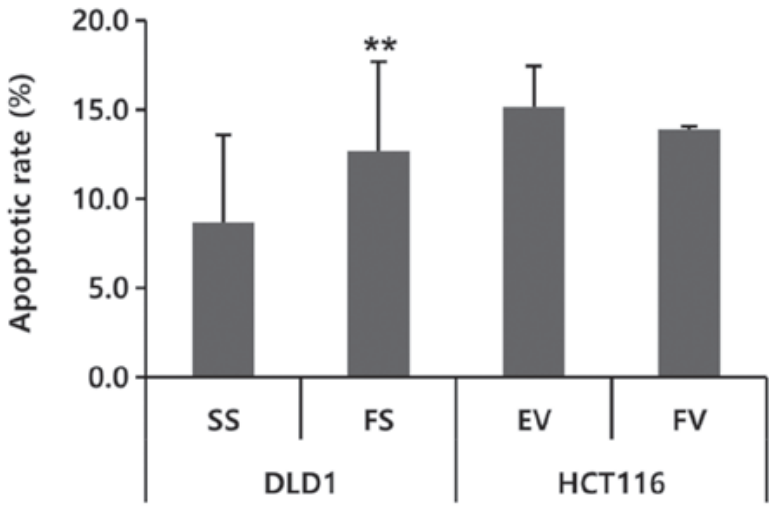

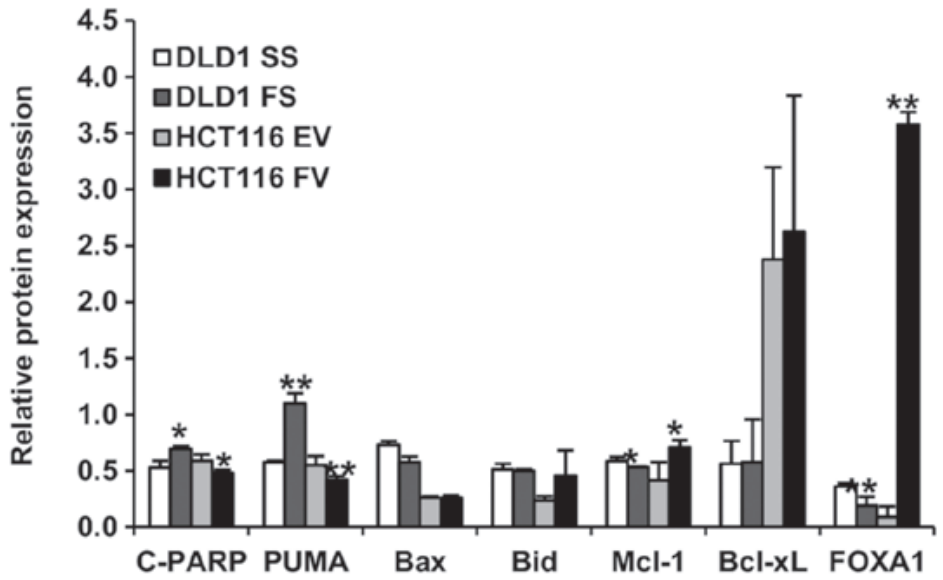

Figure 4. Impact of FOXA1 on apoptosis in human colorectal cancer cells. Flow cytometric analyses and western blotting were performed to evaluate the impact of FOXA1 on apoptosis. (A) Proportion of apoptotic cells was decreased in FV-transfected cells and was increased in FS-transfected cells $\left({ }^{* *} \mathrm{P}<0.01\right)$. (B) Expression levels of cleaved PARP and PUMA were decreased by the overexpression of FOXA1 and increased by FOXA1 knockdown. Expression levels of Bid and Mcl-1 were increased by the overexpression of FOXA1 ( $\mathrm{P}<0.05$ and ${ }^{* *} \mathrm{P}<0.01$, compared to respective control). Bands of the immunoblot were quantified using Multi-Gauge software (ver. 3.0). FOXA1, forkhead box protein A1; siRNA, small interfering RNA; SS, scrambled siRNA; FS, FOXA1 siRNA; EV, empty-pcDNA6-myc vector; FV, pcDNA6-myc-FOXA1 vector, C-PARP; cleaved poly (ADP-ribose) polymerase, PUMA; p53-up-regulated modulator of apoptosis; Bid, BH3 interacting domain death agonist; Mcl-1; myeloid cell leukemia-1.

staining was either absent or weak in the normal colorectal mucosa (Fig. 7A and B). The immunohistochemical staining of FOXA1 protein was predominantly identified in the nucleus of colorectal cancer cells and was not detected in the tumor stroma (Fig. 7C and D). The survival rates of patients with colorectal cancer and the association between the expression of FOXA1 and clinicopathological parameters in these patients were analyzed. It was observed that the expression of FOXA1 was significantly associated with differentiation, cancer stage, lymphovascular invasion, depth of invasion and lymph node metastasis $(\mathrm{P}=0.006,0.001,0.034,0.009$ and 0.002 , respectively; Table I). In addition, the overall survival rates of patients with FOXA1-positive tumors were significantly lower than those of patients with FOXA1-negative tumors ( $\mathrm{P}<0.001$; Fig. 8).

Association between the expression of FOXA1 and cell apoptosis and proliferation in human colorectal cancer.
All tumor samples were subjected to a TUNEL assay and immunohistochemical staining of $\mathrm{Ki}-67$ to determine apoptosis and cell proliferation in the tumor cells. The AI of the 403 tumor samples ranged between 0.9 and 19.9, with a mean AI of $8.7 \pm 6.3$. No significant association was observed between the expression of FOXA1 and AI ( $\mathrm{P}=0.152)$. The KI of the 403 tumor samples ranged between 32.4 and 97.3 , with a mean $\mathrm{KI}$ of $62.4 \pm 18.5$. The mean KI value of FOXA1-positive tumors was 74.8 \pm 14.9 , which was significantly lower than that of FOXA1-negative tumors $(\mathrm{P}<0.001)$ (Table II).

\section{Discussion}

FOXA1 is a member of the forkhead superfamily of transcription factors. FOXA1 is an important regulator in the development, differentiation and metabolism of numerous human organs $(4,5)$. Furthermore, accumulating evidence 
A
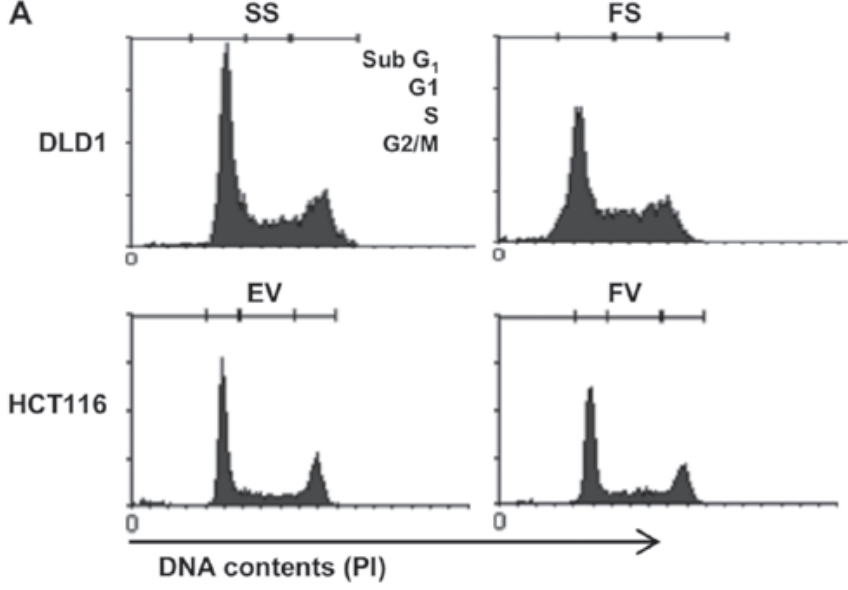

FS

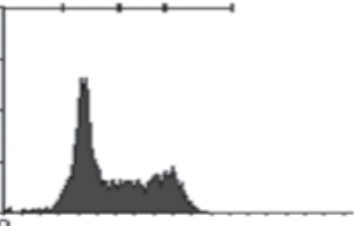

B

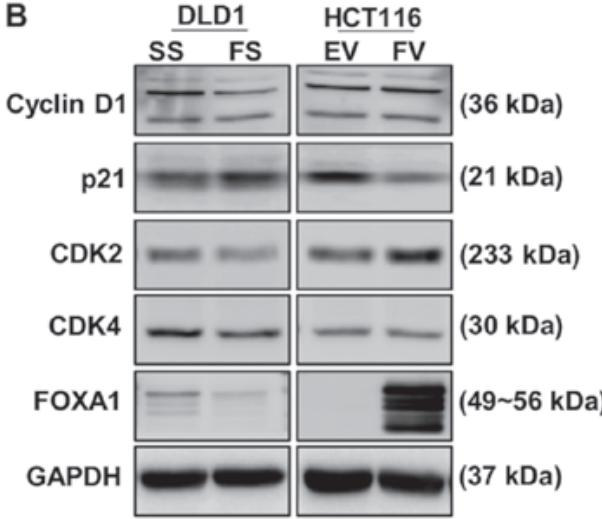

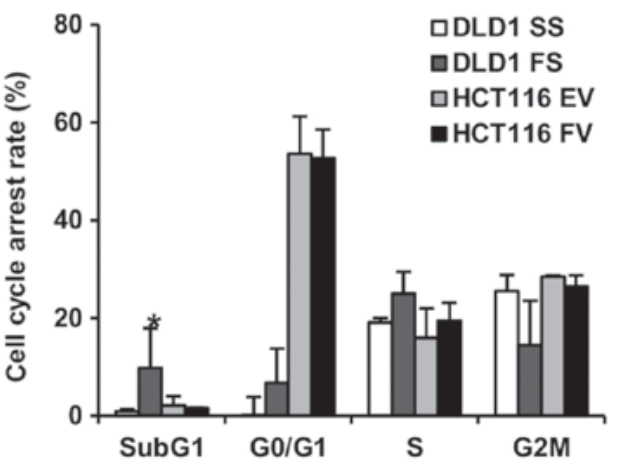

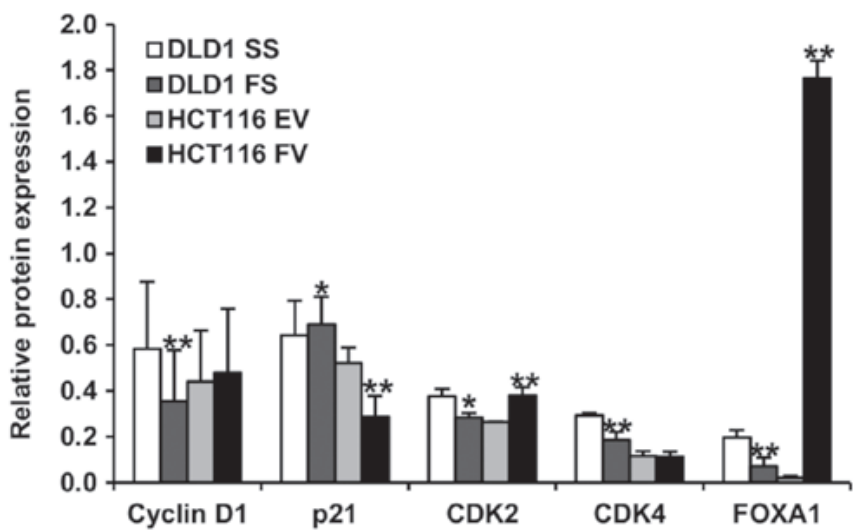

Figure 5. Impact of FOXA1 on cell cycle distribution in human colorectal cancer cells. Flow cytometric analyses and western blotting were performed to evaluate the impact of FOXA1 on cell cycle arrest. (A) Cell cycle analysis demonstrated that FOXA1 knockdown induced cell cycle arrest of the subG1 phase in DLD1 cells ("P<0.05). (B) Protein levels of cyclin D1, CDK2 and CDK4 were significantly decreased by transfection of FS in DLD1 cells. The p21 protein levels were significantly increased by transfection of FS in DLD1 cells ( $\mathrm{P}<0.05$ and ${ }^{* *} \mathrm{P}<0.01$, compared to respective control). Bands of immunoblot were quantified using Multi-Gauge software (ver. 3.0). FOXA1, Forkhead box protein A1; siRNA, small interfering RNA; SS, scrambled siRNA; FS, FOXA1 siRNA; EV, empty-pcDNA6-myc vector; FV, pcDNA6-myc-FOXA1 vector; CDK, cyclin-dependent kinase.
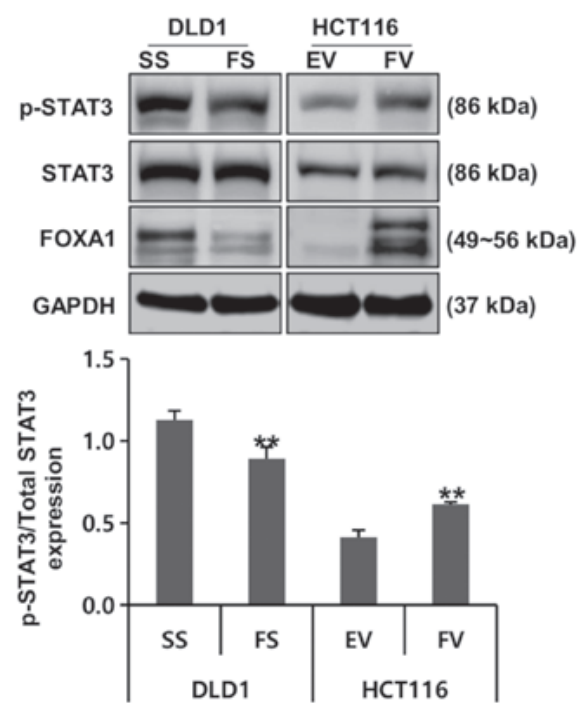

Figure 6. Impact of FOXA1 on oncogenic signaling pathways in human colorectal cancer cells. Phosphorylation of STAT3 was decreased by FOXA1 knockdown. By contrast, phosphorylation of STAT3 was increased by the overexpression of FOXA1 ( $\left({ }^{* *} \mathrm{P}<0.01\right.$, compared to respective control). Bands of the immunoblot were quantified using Multi-Gauge software (ver. 3.0). FOXA1, forkhead box protein A1; siRNA, small interfering RNA; SS, scrambled siRNA; FS, FOXA1 siRNA; EV, empty-pcDNA6-myc vector; FV, pcDNA6-myc-FOXA1 vector, STAT3; signal transducers and activators of transcription 3 ; $\mathrm{p}-$, phosphorylated. has progressively contributed to our understanding of the critical role of FOXA1 in human cancer (6-9). FOXA1 is multifunctional and has been shown to function as a tumor suppressor gene or an oncogene in various types of human cancer (10-23). However, the role of FOXA1 in colorectal cancer lacks support from basic and clinical data.

The regulation of cell migration, invasion and survival is crucial in maintaining normal cellular homeostasis and organogenesis of human tissues. Its loss is a major hallmark of cancer, leading to cancer development and progression (25-27). The present study first investigated the impact of FOXA1 on the alteration of phenotypes in human colorectal cancer cells. Among the human colorectal cancer cells assessed, the protein expression of FOXA1 was the highest in DLD1 cells and the lowest in HCT116 cells. Therefore, siRNA in DLD1 and the pcDNA6-myc vector in HCT116 cells were used to control the endogenous protein expression of FOXA1, either through knockdown or overexpression.

In the present study, FOXA1 knockdown suppressed cell invasion, induced apoptosis and cell cycle arrest and inhibited cell proliferation in human colorectal cancer cells; these effects were reversed following the overexpression of FOXA1. 

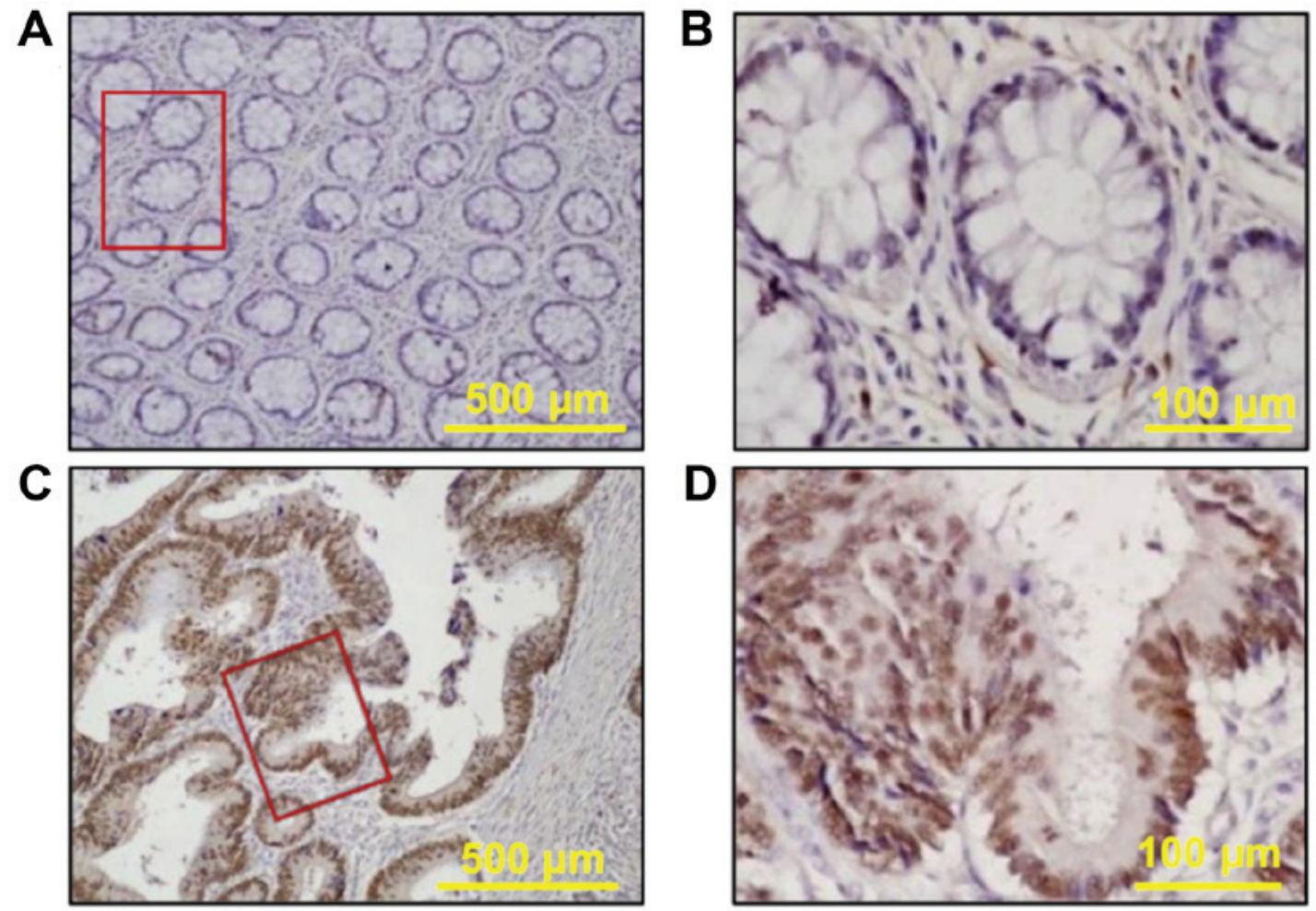

Figure 7. Representative images of immunohistochemical staining for FOXA1. Immunostaining of FOXA1 protein was absent or weak in normal colorectal mucosa. Images at (A) magnification, x100 and (B) magnification, x400). In colorectal cancer tissues, immunostaining of FOXA1 protein at (C) magnification x100 and (D) magnification, $\mathrm{x} 400$ was predominantly observed in the nucleus of cancer cells and not detectable in the tumor stroma FOXA1, forkhead box protein A1.

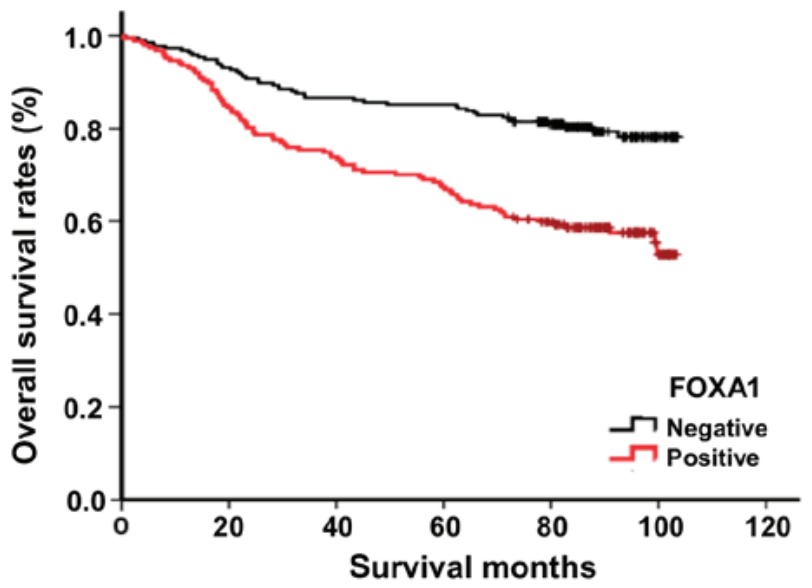

Figure 8. Kaplan-Meier survival curve associating the overall survival with positive expression (dotted line) and negative expression (solid line) of FOXA1 (P<0.01). FOXA1, forkhead box protein A1.

These results suggest that FOXA1 contributes to the alteration of invasive and oncogenic phenotypes in human colorectal cancer cells. In addition, previous studies have indicated that FOXA1 has a potential role in regulating cell migration, invasion and survival in various human cancer cells as an inhibitor or enhancer $(11,15,16,28)$.

To examine the potential mechanisms involved in the above effects, the present study examined the effect of FOXA1 on the stimulation of an oncogenic signaling pathway, involved in cell migration, invasion and cell survival. STAT3 is a key signaling protein that is activated by the stimulation of various cytokines, hormones and growth factors, and elicits diverse biological outcomes including cell growth, differentiation and survival. STAT3 is phosphorylated on its tyrosine residues via Janus kinases, and then forms homo- or heterodimers, translocates to the nucleus, and binds DNA to initiate the transcription of target genes (29-31). The constitutive activation of STAT3 signaling has been reported in numerous human cancer types. However, the regulation and biological consequences of the activation of STAT3 are complex. Aberrant activation of STAT3 has been found to be associated with either oncogenic or tumor suppressing functions in various types of human cancer (32-34). In the present study, the phosphorylation of STAT3 was decreased by FOXA1 knockdown. By contrast, the phosphorylation of STAT3 was increased by the overexpression of FOXA1 in human colorectal cancer cells. These results suggest that FOXA1 may be associated with activation of STAT3, which is important for tumor cell survival in human colorectal cancer.

Subsequently, the expression of FOXA1 was we evaluated in a well-defined series of human colorectal cancer, including long-term and complete follow-up, with specific reference to patient prognosis. It was observed that the expression of FOXA1 was increased in human colorectal cancer tissues compared with that in normal colorectal mucosa. The expression of FOXA1 was significantly associated with cell differentiation, lymphovascular invasion, cancer stage, invasion depth, lymph node metastasis and poor survival rate. Previously, the overexpression of FOXA1 was reported as a good prognostic marker in human estrogen receptor-positive 
Table I. Association between the expression of FOXA1 and the clinicopathological parameters of human colorectal cancer.

\begin{tabular}{|c|c|c|c|c|}
\hline & & FOX & A1 & \\
\hline & Total & Negative & Positive & P-value \\
\hline Parameter & $(n=403)$ & $(n=216)$ & $(n=187)$ & \\
\hline Age (years) & & & & 0.254 \\
\hline$<69.6$ & 176 & 100 & 76 & \\
\hline$\geq 69.6$ & 227 & 116 & 111 & \\
\hline Sex & & & & 0.900 \\
\hline Male & 247 & 133 & 114 & \\
\hline Female & 156 & 83 & 73 & \\
\hline Tumor size (cm) & & & & 0.096 \\
\hline$<4.9$ & 199 & 115 & 84 & \\
\hline$\geq 4.9$ & 204 & 101 & 103 & \\
\hline Histologic type & & & & 0.006 \\
\hline Differentiated & 356 & 182 & 174 & \\
\hline Undifferentiated & 47 & 34 & 13 & \\
\hline Stage & & & & 0.001 \\
\hline I & 63 & 44 & 19 & \\
\hline II & 147 & 86 & 61 & \\
\hline III & 160 & 74 & 86 & \\
\hline IV & 33 & 12 & 21 & \\
\hline Lymphovascular & & & & 0.034 \\
\hline invasion & & & & \\
\hline Negative & 294 & 167 & 127 & \\
\hline Positive & 109 & 49 & 60 & \\
\hline Perineural invasion & & & & 0.170 \\
\hline Negative & 285 & 159 & 126 & \\
\hline Positive & 118 & 57 & 61 & \\
\hline Depth of invasion & & & & 0.009 \\
\hline $\mathrm{T} 1 / \mathrm{T} 2$ & 83 & 55 & 28 & \\
\hline T3/T4 & 320 & 161 & 159 & \\
\hline Lymph node metastasis & & & & 0.002 \\
\hline No & 218 & 132 & 86 & \\
\hline N1-3 & 185 & 84 & 101 & \\
\hline Distant metastasis & & & & 0.248 \\
\hline M0 & 349 & 191 & 158 & \\
\hline M1 & 54 & 25 & 29 & \\
\hline
\end{tabular}

FOXA1, forkhead box protein A1.

breast cancer (11), endometrial (12) and bladder cancer (14), but a poor prognostic indicator in human lung (18), thyroid (19), colorectal (23) and gastric cancer (28). These results suggest that FOXA1 may be a potential prognostic marker, depending on the specific cancer type, and may serve as a poor prognostic marker and a promising therapeutic target in colorectal cancer.

Finally, the present study evaluated the association between the expression of FOXA1 and cell survival,
Table II. Association between the expression of FOXA1 and the apoptosis and proliferation of cells in human colorectal cancer..

\begin{tabular}{lcccr}
\hline & \multicolumn{3}{c}{ FOXA1 expression } & \\
\cline { 3 - 4 } $\begin{array}{l}\text { Parameter } \\
(\text { mean } \pm \text { SD })\end{array}$ & $\begin{array}{c}\text { Total } \\
(\mathrm{n}=403)\end{array}$ & $\begin{array}{c}\text { Negative } \\
(\mathrm{n}=217)\end{array}$ & $\begin{array}{c}\text { Positive } \\
(\mathrm{n}=187)\end{array}$ & P-value \\
\hline AI & $8.7 \pm 6.3$ & $9.8 \pm 7.3$ & $7.2 \pm 4.6$ & 0.152 \\
KI & $62.4 \pm 18.5$ & $53.2 \pm 15.0$ & $74.8 \pm 14.9$ & $<0.001$ \\
\hline
\end{tabular}

FOXA1, forkhead box protein A1; SD, standard deviation; AI, apoptotic index; KI, Ki-67 labeling index.

including proliferation and apoptosis, in human colorectal cancer tissues to confirm the results obtained from the in vitro experiments. It was observed that the mean $\mathrm{KI}$ value of FOXA1-positive tumors was significantly higher than that of FOXA1-negative tumors. Ki-67 is a nuclear antigen and an established proliferation marker of tumor cells in various types of human cancer, including colorectal cancer (35-37). However, no significant association was observed between the expression of FOXA1 and the AI value. These in vivo results are in accordance with the conclusion that FOXA1 serves a crucial role in cell proliferation in human colorectal cancer cell lines.

In conclusion, FOXA1 is an important mediator of proliferative and anti-apoptotic activities in human colorectal cancer cells. FOXA1 was upregulated in human colorectal cancer tissues and was associated with poor prognosis, suggesting an oncogenic role of FOXA1 in the development and progression of human colorectal cancer.

\section{Acknowledgements}

Not applicable.

\section{Funding}

This study was supported by research funds from the Research Institute of Clinical Medicine, Chonnam National University Hwasun Hospital in 2017 (grant no. HCRI 17912-1), Republic of Korea, and the National Research Foundation of Korea grant (grant no. NRF-2017R1A2B4004703) funded by the Korean government (Ministry of Science, ICT and Future Planning), Republic of Korea.

\section{Availability of data and materials}

All data generated or analyzed during this study are included in this published article.

\section{Authors' contributions}

YEJ was involved in the conceptualization of the study. YLP, SHK, SYP, MWJ, SYH, JHC, DSM, SBC, WSL and HSK were involved in data curation, were responsible for formal analysis 
and provided resources. YLP, SHK and YEJ were involved in the investigative part of the study. YEJ was involved in the methodology, was involved in project administration and was involved in the writing of the manuscript and original draft preparation. YLP, SHK and YEJ were involved in the writing, reviewing and editing of the manuscript. All authors read and approved the final manuscript

\section{Ethics approval and consent to participate}

The present study was approved by the Institutional Review Board of the Chonnam National University Hwasun Hospital (Jeonnam, Korea; CNUHH-2017-163). This was a retrospective study and written informed consent was obtained from each participant prior to tissue acquisition at the time of hospitalization. All participants provided written consent for their information to be stored in the hospital database and used for research.

\section{Patient consent for publication}

Not applicable.

\section{Competing interests}

The authors declare that they have no competing interests.

\section{References}

1. Brenner H, Kloor M and Pox CP: Colorectal cancer. Lancet 383: 1490-1502, 2014.

2. Choi Y, Sateia HF, Peairs KS and Stewart RW: Screening for colorectal cancer. Semin Oncol 44: 34-44, 2017.

3. Ahmed FE: Development of novel diagnostic and prognostic molecular markers for sporadic colon cancer. Expert Rev Mol Diagn 5: 337-352, 2005

4. Katoh M and Katoh M: Human FOX gene family (Review). Int J Oncol 25: 1495-1500, 2004.

5. Kaestner KH: The FoxA factors in organogenesis and differentiation. Curr Opin Genet Dev 20: 527-532, 2010.

6. Bernardo GM and Keri RA: FOXA1: A transcription factor with parallel functions in development and cancer. Biosci Rep 32: 113-130, 2012.

7. Augello MA, Hickey TE and Knudsen KE: FOXA1: Master of steroid receptor function in cancer. EMBO J 30: 3885-3894, 2011.

8. Katoh M, Igarashi M, Fukuda H, Nakagama $\mathrm{H}$ and Katoh M: Cancer genetics and genomics of human FOX family genes. Cancer Lett 328: 198-206, 2013.

9. Zhang G, Zhao Y, Liu Y, Kao LP, Wang X, Skerry B and Li Z: FOXA1 defines cancer cell specificity. Sci Adv 2: e1501473, 2016.

10. Shou J, Lai Y, Xu J and Huang J: Prognostic value of FOXA1 in breast cancer: A systematic review and meta-analysis. Breast 27: 35-43, 2016.

11. Park S, Koh E, Koo JS, Kim SI, Park BW and Kim KS: Lack of both androgen receptor and forkhead box A1 (FOXA1) expression is a poor prognostic factor in estrogen receptor-positive breast cancers. Oncotarget 8: 82940-82955, 2017.

12. Abe Y, Ijichi N, Ikeda K, Kayano H, Horie-Inoue K, Takeda S and Inoue S: Forkhead box transcription factor, forkhead box A1, shows negative association with lymph node status in endometrial cancer, and represses cell proliferation and migration of endometrial cancer cells. Cancer Sci 103: 806-812, 2012.

13. Yamashita H, Amponsa VO, Warrick JI, Zheng Z, Clark PE, Raman JD, Wu XR, Mendelsohn C and DeGraff DJ: On a FOX hunt: Functions of FOX transcriptional regulators in bladder cancer. Nat Rev Urol 14: 98-106, 2017.
14. Reddy OL, Cates JM, Gellert LL, Crist HS, Yang Z, Yamashita H, Taylor JA III, Smith JA Jr, Chang SS, Cookson MS, et al: Loss of FOXA1 drives sexually dimorphic changes in urothelial differentiation and is an independent predictor of poor prognosis in bladder cancer. Am J Pathol 185: 1385-1395, 2015.

15. Gan HY, Li N, Zhang Q and Feng ZZ: Silencing FOXA1 gene regulates liver cancer cell apoptosis and cell proliferation. Eur Rev Med Pharmacol Sci 22: 397-404, 2018.

16. He S, Zhang J, Zhang W, Chen F and Luo R: FOXA1 inhibits hepatocellular carcinoma progression by suppressing PIK3R1 expression in male patients. J Exp Clin Cancer Res 36: 175, 2017.

17. Song Y, Washington MK and Crawford HC: Loss of FOXA1/2 is essential for the epithelial-to-mesenchymal transition in pancreatic cancer. Cancer Res 70: 2115-2125, 2010.

18. Li J, Zhang S, Zhu L and Ma S: Role of transcription factor FOXA1 in non-small cell lung cancer. Mol Med Rep 17: 509-521, 2018.

19. Li M, Zhang W, Liu C, Shi Y, Tang W, Chen S, Gu H, Yin J, Zhang Z and Jiang P: Forkhead box A1 (FOXA1) tagging polymorphisms and esophageal cancer risk in a Chinese population: A fine-mapping study. Biomarkers 21: 523-529, 2016.

20. Tsourlakis MC, Eleftheriadou A, Stender A, Weigand P, Grupp K, Hube-Magg C, Kluth M, Schroeder C, Steurer S, Hinsch A, et al: FOXA1 expression is a strong independent predictor of early PSA recurrence in ERG negative prostate cancers treated by radical prostatectomy. Carcinogenesis 38: 1180-1187, 2017.

21. Takayama K and Inoue S: Transcriptional network of androgen receptor in prostate cancer progression. Int J Urol 20: 756-768, 2013.

22. Nucera C, Eeckhoute J, Finn S, Carroll JS, Ligon AH, Priolo C, Fadda G, Toner M, Sheils O, Attard M, et al: FOXA1 is a potential oncogene in anaplastic thyroid carcinoma. Clin Cancer Res 15: 3680-3689, 2009.

23. Ma W, Jiang J, Li M, Wang H, Zhang H, He X, Huang L and Zhou Q: The clinical significance of forkhead box protein A1 and its role in colorectal cancer. Mol Med Rep 14: 2625-2631, 2016.

24. Greene FL, Page DL, Fleming ID, Fritz AG, Balch CM, Haller DG and Morrow M: AJCC cancer staging manual. 6th edition. Springer-Verlag, New York, NY, 2002.

25. Chambers AF, Groom AC and MacDonald IC: Dissemination and growth of cancer cells in metastatic sites. Nat Rev Cancer 2: 563-572, 2002.

26. Brábek J, Mierke CT, Rösel D, Veselý P and Fabry B: The role of the tissue microenvironment in the regulation of cancer cell motility and invasion. Cell Commun Signal 8: 22, 2010.

27. Kiechle FL and Zhang X: Apoptosis: Biochemical aspects and clinical implications. Clin Chim Acta 326: 27-45, 2002.

28. Ren H, Zhang P, Tang Y, Wu M and Zhang W: Forkhead box protein A1 is a prognostic predictor and promotes tumor growth of gastric cancer. Onco Targets Ther 8: 3029-3039, 2015.

29. Villarino AV, Kanno Y, Ferdinand JR and O'Shea JJ: Mechanisms of Jak/STAT signaling in immunity and disease. J Immunol 194: 21-27, 2015.

30. Abroun S, Saki N, Ahmadvand M, Asghari F, Salari F and Rahim F: STATs: An old Story, yet mesmerizing. Cell J 17: 395-411, 2015.

31. Zundler S and Neurath MF: Integrating immunologic signaling networks: The JAK/STAT pathway in colitis and colitis-associated cancer. Vaccines (Basel) 4: E5, 2016.

32. Guanizo AC, Fernando CD, Garama DJ and Gough DJ: STAT3: A multifaceted oncoprotein. Growth Factors 36: 1-14, 2018.

33. Aigner P, Just V and Stoiber D: STAT3 isoforms: Alternative fates in cancer? Cytokine Jul 18, 2018 (Epub ahead of print). pii: S1043-4666(18)30300-4,2018.doi: 10.1016/j.cyto.2018.07.014.

34. Lai PS, Rosa DA, Magdy Ali A, Gómez-Biagi RF, Ball DP, Shouksmith AE and Gunning PT: A STAT inhibitor patent review: Progress since 2011. Expert Opin Ther Pat 25: 1397-1421, 2015.

35. Sun $X$ and Kaufman PD: Ki-67: More than a proliferation marker. Chromosoma 127: 175-186, 2018.

36. Ragab HM, Samy N, Afify M, El Maksoud NA and Shaaban HM: Assessment of $\mathrm{Ki}-67$ as a potential biomarker in patients with breast cancer. J Genet Eng Biotechnol 16: 479-484, 2018.

37. Hilska M, Collan YU, O Laine VJ, Kössi J, Hirsimäki P, Laato M and Roberts PJ: The significance of tumor markers for proliferation and apoptosis in predicting survival in colorectal cancer. Dis Colon Rectum 48: 2197-2208, 2005. 